\title{
Effect of preoperative forced-air warming (PREWARM) on intraoperative tissue oxygenation, microperfusion and body core temperature in patients with ovarian cancer undergoing major cytoreductive surgery
}

\author{
Kaufner L, Niggemann P, Boschmann M, Sehouli J, Spies CD, von Heymann C
}

Charité-Universitätsmedizin Berlin, Department of Anesthesiology and Operative Intensive Care Medicine, Campus Virchow Klinikum/Campus Charité Mitte, Berlin, Germany

Background: Cytoreductive surgery is associated with extensive fluid resuscitation, inadvertent periop. hypothermia and high dose usage of vasopressors which may alter intraop. tissue oxygenation $\left(\mathrm{StO}_{2}\right)$, microperfusion and subsequently reduce postop. outcome. We hypothesize that preop. forced-air warming (PW) may help to prevent intraop. hypothermia as a prerequisite to improve $\mathrm{StO}_{2}$ and microperfusion.

Material and Methods: After ethics approval 47 women scheduled to have cytoreductive surgery were prospectively enrolled. All women received a thoracic PDA, an arterial line, a central venous catheter during induction of GA and intraop. forced-air warming. According to their randomization women were treated with either $30 \mathrm{~min}$. of $43^{\circ} \mathrm{C}$ PW (Gr. 1, Fig 1A), PW + subcutaneous microdialysis (MD, Gr. 2, Fig 1B), MD without $\mathrm{PW}$ (st/MD, Gr. 3) or no intervention (st., Gr. 4). StO2, central venous-arterial carbon dioxide difference $\left(\mathrm{dCO}_{2}\right)$ and ethanol ratio via $\mathrm{MD}$ for microperfusion and core temp. (hourly) were defined as primary outcome. Additionally, hemodynamic parameters (MAP, CVP, HF, norepinephrine) and glucose, lactate and glycerol in MD were obtained. Statistical analysis was performed using the Mann-Whitney-U-Test and nonparametric-longitudinal analysis.

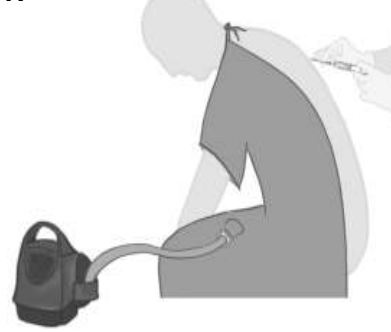

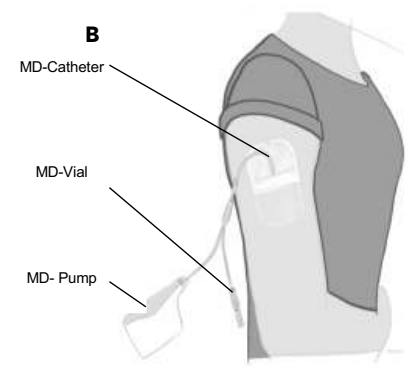

Fig.1: A) Forced Air Prewarming during PDA; B) Subcutaneous Microdialysis (MD);

Results: No significant differences in $\mathrm{StO}_{2}$ of $86.0 \%$ (84.0-88.0) in Gr. $1+2(n=24)$ compared to $84.0 \%(80.0-87.5)$ in Gr. $3+4$ $(n=23)$ were detectable at $60 \mathrm{~min}$ and over the entire intraop. Period (Fig. 2). The $\mathrm{dCO}_{2}$ at $60 \mathrm{~min}(\mathrm{Gr} .1+2: 6.3 \mathrm{mmHg}(5.4-$ 7.8) vs. Gr. $3+4: 5.7 \mathrm{mmHg}(4.7-6.9))$ and over the entire intraop. period remains without significant differences.

In MD the ethanol ratio in $\mathrm{Gr} .2(n=7)$ is fairly constant at 0.4 compared to a slight decrease from 0.45 to 0.25 in Gr. $3(n=7)$. Lactate, glucose and glycerol in Gr. 2 tend to be more constant over the entire period without significant differences to Gr. 3 (Fig.3)
The median core temp. of $36.7^{\circ} \mathrm{C}(36.6-36.9)$ in $\mathrm{Gr} .1+2$ at $60 \mathrm{~min}$ was significantly $(p<0.001)$ increased compared to $35.8^{\circ} \mathrm{C}$ (35.5 - 36.2) in Gr. 3+4 (Fig. 4). A significant intraop. temperature gap of $0.8^{\circ} \mathrm{C}(p<0.001)$ between both groups was registered in. Hemodynamic parameters showed no significant differences between the groups.

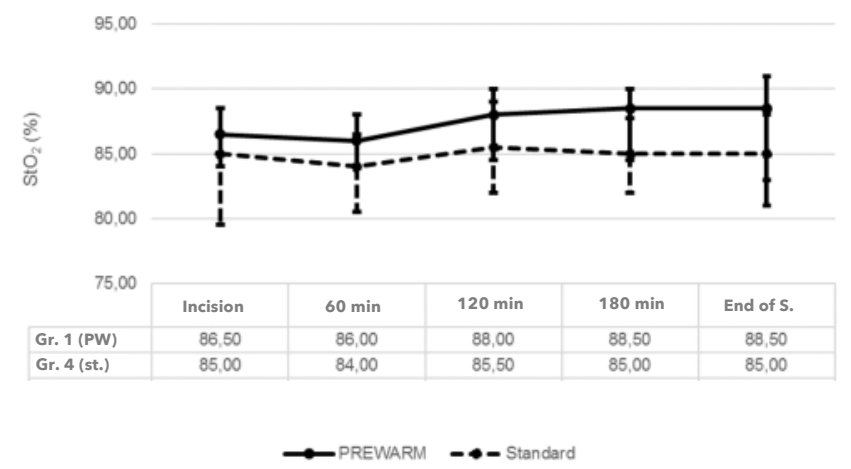

Fig.2: Tissue Oxygenation (median/quartil: 25\%; 75\%)
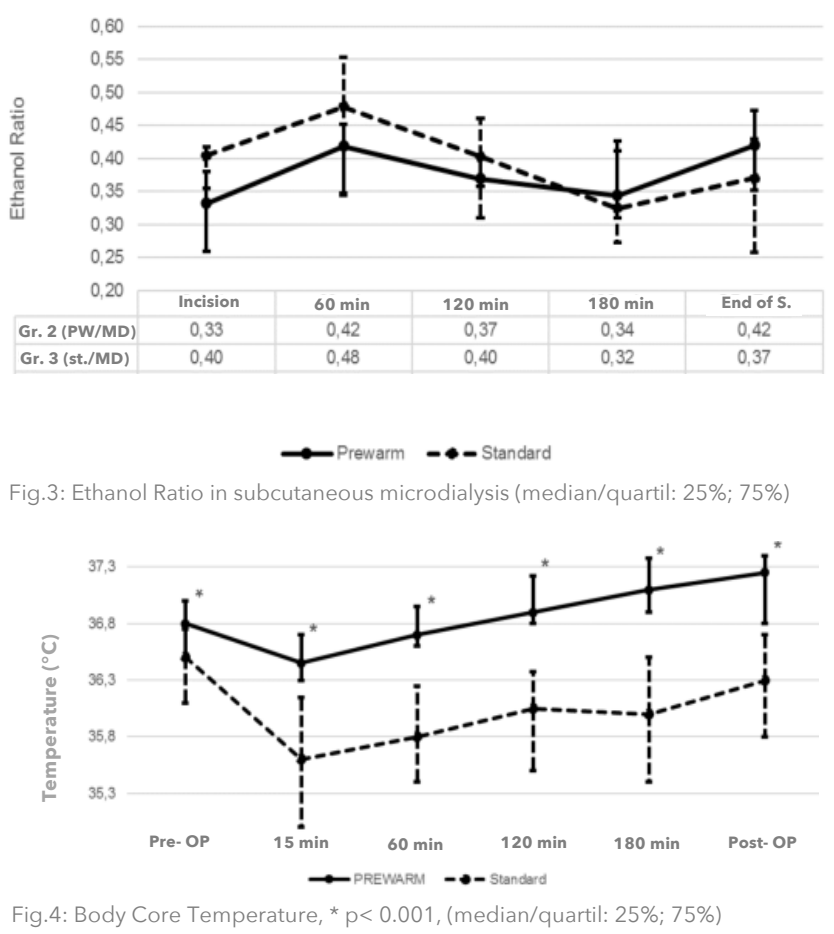

Conclusions: No effect in $\mathrm{StO}_{2}$ and microperfusion as function of PW is detectable. Nevertheless, MD parameters should provide further information on stabilized intraop. microperfusion in normothermic patients. $\mathrm{PW}$ in order to maintain normothermia in cytoreductive surgery is effective. 